\title{
Impact of excess TGF $\beta$ on bone and muscle in condition of diet-induced obesity in mice with Camurati-Engelmann Disease
}

\author{
Asma S. Bahrami ${ }^{1}$, Trupti Trivedi ${ }^{2}$, Gabriel M. Pagnotti ${ }^{2}$, Khalid M. Mohammad ${ }^{2}$, \\ Theresa A. Guise ${ }^{2}$ \\ ${ }^{1}$ Indiana University School of Medicine, ${ }^{2}$ Division of Endocrinology, Department \\ of Medicine; Indiana University School of Medicine; Indianapolis, Indiana \\ Background and Hypothesis: Camurati-Engelmann Disease (CED) is \\ characterized by extreme bone turnover and excess TGF- $\beta$ release. We \\ previously showed that bone-derived TGF- $\beta$ causes glucose intolerance, \\ increases skeletal muscle weakness, and exacerbates diet-induced obesity in \\ CED mice. However, it is unknown whether glucose intolerance and obesity alter \\ bone and muscle phenotypes. Thus, we hypothesized that impaired glucose \\ metabolism and diet-induced obesity exacerbate bone and muscle loss in a \\ mouse model of CED.
}

Experimental Design: 45-week WT and CED mice were fed either high-fat diet (HFD) or low-fat diet (LFD) for 15 weeks. Ex vivo bone micro-CT and histomorphometry were used to evaluate bone and muscle. Statistical analysis was performed using GraphPad Prism with $p<0.05$ considered significant.

Results: CED mice showed severe cortical and trabecular bone loss in response to diet-induced obesity. Trabecular bone volume was reduced by $37 \%$ in L5 vertebrae $(p<0.001), 16 \%$ in tibiae $(p<0.05)$, and $7 \%$ in femora in CED-HFD compared to WT-HFD. Bone mineral density was reduced $(p<0.0001)$ and cortical porosity was increased $(p<0.0001)$ in CED-HFD vs WT-HFD in femora and tibiae. Bone histomorphometry showed no significant differences in osteoclast number between groups. pSMAD2/3 staining was increased by $25 \%$ $(p<0.05)$ and muscle fiber diameter was reduced by $32 \%(p<0.05)$ in the tibialis anterior muscle of CED mice compared to WT, with greater changes in HFD-fed mice.

Conclusion and Potential Impact: High-fat diet and impaired glucose metabolism exacerbates bone loss and increases TGF- $\beta$ signaling in CED mice. In future studies, inhibiting TGF- $\beta$ signaling and reducing adiposity may prevent glucose intolerance and musculoskeletal deterioration in conditions of high bone turnover. 